\title{
Percepción espacio-temporal en la obra de Samuel Beckett y Alberto Giacometti
}

\section{Space-Time Perception in the Works of Samuel Beckett and Alberto Giacometti}

Artículo recibido el 5 de abril de 2016; devuelto para revisión el 23 de noviembre de 2016; aceptado el I5 de diciembre de 20I6. http:dx.doi.org/I0.22207/iie.I8703062e.2017.I.2590.

María del Carmen

Molina Barea

Líneas de investigación

Lines of research

Publicaciones más relevantes
Universidad de Córdoba, Facultad de Filosofía y Letras, Departamento de Ciencias Sociales y Humanidades, España, mcpalladio@ hotmail.com.

Estética y teoría del arte contemporáneo; surrealismo; cultura visual; filosofía del cine.

Contemporary esthetics and art theory; surrealism; visual culture; philosophy of cinema.

"Paprika (Satoshi Kon, 2006): Animación japonesa desde el surrealismo", El Respirador. Revista de Arte Contemporáneo, núm. 4 (2016): 34-45; "Literatura menor y resistencia biopolítica en la obra de Jesús Lizano", Metáforas de la Multitud (2016): 325-339; "La identidad del militante artístico: el caso de Joseph Beuys", ARTS. Revista de la Feria Internacional de Arte Contemporáneo de Sevilla, núm. I (20I5): 8085; "Ese ojo sin moral: las repercusiones estéticas del cine como ojo mecánico desde el ultraísmo al surrealismo", De arte. Revista de Historia del Arte, núm. I3 (2014): 192-2I5; "Buster Keaton y el surrealismo de la Residencia de Estudiantes. Razones de una confluencia", Archivo Español de Arte, núm. 34I (2013): 29-48; "Sexo y destrucción en el surrealismo español: Buñuel, Dalí y Lorca según la pulsión de muerte", Anales de Historia del Arte, núm. 22 (2012): 167-192; "Dalí y la Hypnerotomachia Poliphili”, Cuadernos de Arte e Iconografía, núm. 42 (2012): 355-409.

Resumen El presente artículo plantea un análisis de la percepción espacio-temporal en la producción artística de Samuel Beckett y Alberto Giacometti. Se tomarán como objeto de estudio dos obras concretas: por un lado, el montaje televisivo Quad I \& II, de Beckett, y por otro, el texto titulado "El sueño, el Sphinx y la muerte de T.", escrito por Giacometti. Ambas piezas se abordarán desde el concepto de tiempo de 
Henri Bergson, la teoría del universo tetradimensional de Hermann Minkowski, y la visión esquizofrénica del espacio-tiempo según Eugène Minkowski.

Palabras clave Beckett; Giacometti; Bergson, Minkowski; espacio; tiempo; esquizofrenia.

Abstract The present paper provides an analysis of the space-time perception within the artistic production of Samuel Beckett and Alberto Giacometti. It focuses on two works in particular: on the one hand, Beckett's TV play Quad I \& II, and on the other, the text entitled "The Dream, the Sphinx, and the Death of T.", written by Giacometti. Both works will be studied according to Henri Bergson's notion of time, Hermann Minkowski's theory of a four-dimensional universe, and the schizophrenic view of space-time developed by Eugène Minkowski.

Keywords Beckett; Giacometti; Bergson; Minkowski; space; time; schizophrenia. 
DOI: http://dx.doi.org/10.22201/iie.18703062e.2017.1.2590

\title{
MARÍA DEL CARMEN MOLINA BAREA \\ UNIVERSIDAD DE CÓRDOBA,
} FACULTAD DE FILOSOFÍA Y LETRAS

DEPARTAMENTO DE CIENCIAS SOCIALES Y HUMANIDADES, ESPAÑA

\section{Percepción espacio-temporal en la obra de Samuel Beckett y Alberto Giacometti}

\author{
Beckett, Giacometti y la influencia de Bergson
}

¿Les interesa el espacio? Hagamos que cruja. ¿Les atormenta el tiempo? Matémoslo juntos. ${ }^{1}$

\begin{abstract}
unque no en exceso conocida, la amistad entre Samuel Beckett y $A$ Alberto Giacometti conforma una de las relaciones de intercambio 1 y compañerismo más duraderas de entre las surgidas al amparo de las vanguardias en el París de entreguerras. Ambos artistas confluyen en este escenario, uno procedente de Irlanda, el otro de Suiza, y los une una incontestable afinidad intelectual, traducida a lo largo de los ańos en fuente de inspiración recíproca. De ésta ha quedado como testimonio la colaboración del dramaturgo y el escultor en la realización del decorado de En attendant Godot (I953), con ocasión de su reposición en 196r en el Teatro Odéon de París.

Amigos de cabarets y paseos nocturnos, Beckett y Giacometti se conocen hacia 1937, participan del ambiente parisino en la época de esplendor del

I. Samuel Beckett, Disjecta. Escritos misceláneos y un fragmento dramático, trad. Alicia Martínez Yuste (Madrid: Arena Libros, 2009), I45.
\end{abstract}


surrealismo, movimiento artístico encabezado por el poeta André Breton, en cuyas lides se integra el joven Giacometti. ${ }^{2}$ Por su parte, Beckett mantiene también cierto contacto con el surrealismo, llega incluso a traducir la obra de algunos de sus representantes más destacados, como Paul Éluard, René Crevel y el propio Breton. Ese París de los ańos treinta, jalonado por la efervescente actividad de las vanguardias, conoce asimismo un momento especialmente favorable a la difusión y recepción del pensamiento de Henri Bergson, filósofo francés que en 1927 fue galardonado con el Premio Nobel de Literatura. Por ejemplo, resulta interesante comprobar las numerosas referencias de André Breton a Bergson, o las alusiones de otros autores surrealistas, como Antonin Artaud, quien cita al filósofo en el artículo "L'homme contre le destin". ${ }^{3}$

Ciertamente, cabe deducir una considerable influencia bergsoniana en los modos estéticos del surrealismo. Libros de Bergson como Matière et mémoire (1896) y L'énergie spirituelle (1919), basado en una conferencia de I9or titulada Le rêve, dejan su huella en el imaginario surrealista: desde el concepto de memoria al de creatividad, sin olvidar el interés por el sueño y la paramnesia (ilusión de lo ya vivido). Pero por encima de todo, la filosofía de Bergson determina cierta actitud subversiva frente a la idea tradicional, fija e inamovible, de tiempo y espacio, ahora entendida como "duración (durée)" e "intuición". ${ }^{4}$ De esto se hacen eco rápidamente los artistas del surrealismo. Como dijera Jack Spector: "Los surrealistas, enemigos de las continuidades temporales y espaciales, a las que consideraban aliadas del racionalismo burgués, dieron la bienveni-

2. Anne Atik recuerda con humor a su marido, el pintor Avigdor Arikha, junto a Samuel Beckett, gran amigo del matrimonio, en sus paseos nocturnos por París, "camino del Dôme, en donde solían toparse con o esquivar a Alberto Giacometti (debido a que, cada vez que los veía, siempre les soltaba la misma, aunque singular, historia acerca de la revelación que tuvo mientras estaba sentado en el cine Cinéac, en una época en que le costaba percibir y dibujar)", en Anne Atik, Cómo fue. Recuerdos de Samuel Beckett, trad. Juan Abeleira (Barcelona: Circe, 2005), I8.

3. Antonin Artaud, ("Homme contre le destin" [1936], en Messages révolutionaires (París: Gallimard, 1971), 24-34.

4. "La duración comporta, en su misma estructura, una radical exigencia de heterogeneidad. [...] La heterogeneidad es el ámbito de la pura cualidad, de la diferencia, del policentrismo, de la ausencia de referencias privilegiadas que puedan ordenar conjuntos homogéneos. Es el puro reino de la dispersión, de la originalidad”, en Ignacio Izuzquiza Otero, Henri Bergson: la arquitectura del deseo (Universidad de Zaragoza, 1986), 39. Asimismo: "La intuición es la facultad que permite captar la duración de un modo completo y eficaz. [...] Precisamente porque la intuición tiene una estructura semejante a la duración y es ella misma proceso, puede captar directamente, sin intermediario de espacio y lenguaje, el movimiento mismo, el proceso dinámico que constituye la duración y, al mismo tiempo, constituye la base de la realidad", en Izuzquiza Otero, Henri Bergson, 245. 
da a todos los que, en la ciencia o en las artes, atacaban las ideas convencionales de tiempo y espacio."s

Inmersos en este clima de impronta bergsoniana, no sólo los surrealistas reciben su influencia, entre ellos Giacometti, sino también Beckett. Como indica Manfred Milz, Samuel Beckett adquiere familiaridad con la filosofía de Bergson en el Trinity College de Dublín y en l'École Normale Supérieure de París. En concreto, llama la atención que A. A. Luce, reconocido experto en el pensamiento de Bergson, fuera el tutor de Beckett en el Trinity College entre I923 y $1927 .{ }^{6}$ Después, en la etapa de l'École Normale Supérieure, Beckett se empapa de la teoría de la temporalidad de Bergson y consagra un verano entero a la lectura de $\dot{A}$ la recherche $d u$ temps perdu (I9I3-1927).7 Como resultado de esta incursión en la obra maestra de Marcel Proust, Beckett publica en I93I sus reflexiones sobre el tiempo en forma de un ensayo titulado precisamente Proust. ${ }^{8}$ Así las cosas, no es casualidad que se haya señalado repetidas veces la problemática temporal latente en las obras de Beckett. Hasta tal punto que se podría afirmar que "el Tiempo es el único personaje que 'pasa' por la escena beckettiana y el que, desde bastidores, ha marcado previamente los pasos y el destino de los demás"?

5. Jack Spector, Arte y escritura surrealistas (I9I9-1939). El oro del tiempo (Madrid: Síntesis, 1997), 62.

6. Manfred Milz, Samuel Beckett und Alberto Giacometti. Das Innere als Oberfläche; ein ästhetischer Dialog im Zeichen schöpferischer Entzweigungsprozesse (1929-1936) (Würzburg: Königshausen \& Neumann, 2006), 258.

7. "Beckett's analysis of the Proustian aesthetic is centred around an account of the working of habit upon memory, and the consequences these have for the subjective experience of time". ("El análisis de Beckett acerca de la estética de Proust se centra en una consideración del funcionamiento del hábito sobre la memoria, y en las consecuencias que ello tiene en la experiencia subjetiva del tiempo.", en John Pilling, ed., The Cambridge Companion to Beckett [Cambridge University Press, 2006], 4) (trad. de la autora).

8. "Proust, as seen by Beckett, divides time up into segments, each representing a change in the personality and mental growth of the individual, corresponding to a change in his personality and intelectual development. This, in turn, corresponds to a change in his habit formation, or more exactly of the contractual arrangement between the individual mind and the external world." ("Proust, según la visión de Beckett, divide el tiempo en fragmentos, representando cada uno de ellos un cambio en la personalidad y crecimiento mental del individuo, correspondiéndose con un cambio en su personalidad y desarrollo intelectual. Esto, a su vez, se corresponde con un cambio en la formación de su hábito, o más concretamente, en el acuerdo contractual entre la mente individual y el mundo externo", en John Calder, The Philosophy of Samuel Beckett [Londres: Calder Publications London/Riverrun Press New Jersey, 200I], 65-66) (trad. de la autora).

9. Juan Bargalló Carraté y Francisco García Tortosa, eds., Samuel Beckett: palabra y silencio (Sevilla: Centro Andaluz de Teatro, Padilla Libros, I99I), I. 
DOI: http://dx.doi.org/10.22201/iie.18703062e.2017.1.2590

\section{Beckett y el espacio tetradimensional}

Porque la estructura del espacio jes forzosamente invariable en el tiempo? Ciertamente no lo es. ${ }^{\text {Io }}$

Interesado no sólo en la problemática temporal, Samuel Beckett dialoga también con el pensamiento bergsoniano en el intento de aclarar su propia concepción del espacio. ${ }^{\text {II }}$ De hecho, la preocupación de Beckett por este tema continúa hasta finales de los años cuarenta, época en la que sostiene acalorados debates con Georges Duthuit, crítico de arte francés muy apegado a los surrealistas y ferviente seguidor de Bergson. Si bien la obra que mejor ejemplifica el acercamiento beckettiano al problema del espacio data de 1980 . Se trata de una pequeńa pieza de teatro mudo, reconvertida en dos versiones para ser emitidas por televisión: la primera a color, la segunda en blanco y negro, de título Quad I y Quad II.

En Quad hallamos un minucioso estudio de las posibilidades que ofrece el espacio por medio de un restringido ejercicio de movimiento. En la obra aparecen cuatro personajes no identificados, vestidos con túnicas y capuchas, que recuerdan vagamente la imagen de un monje. Estas figuras se mueven en un espacio geométrico limitado, sobre un tapete cuadrado dispuesto en el suelo. Desde cada uno de los vértices, los personajes van entrando en el cuadrado de manera escalonada, como si se tratara de una fuga musical. Una vez dentro, recorren los lados del cuadrado y, llegados a un vértice, se aproximan, en diagonal, al centro del espacio. Desde allí rotan la dirección y se dirigen hacia otro de los vértices, así sucesivamente hasta que agotan todos los itinerarios (fig. I). Los personajes, por tanto, nunca cruzan sus trayectorias, y progresivamente abandonan el cuadrado por el último vértice, una vez que han consumido todos los recorridos posibles. ${ }^{12}$ La pieza presenta un ritual de movimientos erráticos, casi una danza de precisión y coordinación reducida a sus mínimos elementos, que recuerda hasta cierto punto al performance de Esther Ferrer Recorrer un cuadrado de todas las formas posibles (1997).

I0. Xavier Zubiri, Espacio. Tiempo. Materia (Madrid: Fundación Xavier Zubiri/Alianza, 1996), 106.

II. Stephen Elliot Wilmer y Audrone Žukauskaite, eds., Deleuze and Beckett (Londres y Nueva York: Palgrave Macmillan, 20I5), I44.

I2. H. H. Hiebel, "Quadrat I +2 as Television Play", en Marius Buning y Lois Oppenheim eds., Beckett in the Igoos, Samuel Beckett Today/Aujourd'hui (Ámsterdam y Atlanta: Rodopi, 1993), 335-342. 
I. Diagrama secuencial de Quad I \& Quad II (ilustración de la autora basada en el original de Samuel Beckett).

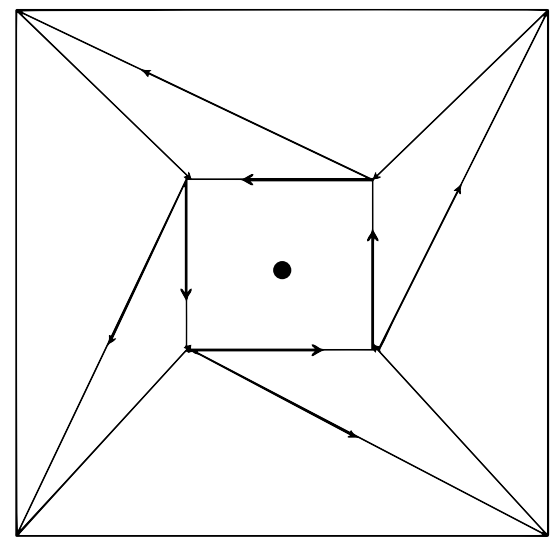

Quad se constituye en una repetición rítmica en bucle, emparentada con lo que Gilles Deleuze y Félix Guattari han denominado "estribillo" o "ritornelo", es decir, una repetición de lo mismo que, al repetirse, difiere cada vez. Tanto es así que el propio Deleuze reflexiona expresamente sobre esta pieza de Beckett en Critique et clinique (1993) y en el ensayo titulado L'Épuisé (1992). ${ }^{\mathrm{I3}}$ Deleuze incide especialmente en el agotamiento de las posibilidades espaciales que se pone de manifiesto en Quad. ${ }^{I 4}$ Asimismo, destaca una nueva gramática visual en la obra filmada de Beckett, que denomina lengua III, capaz de producir una imagen en si misma basada en el ritornelo. ${ }^{15}$ Quad está filmado con cámara fija, situada desde arriba, sin cortes de montaje, lo cual genera la sensación de un

13. Samuel Beckett, Quad et autres pièces pour la télévision suivi de Gilles Deleuze, L'épuisé (París: Les Éditions de Minuit, 1992).

I4. 'L'épuisé parte de la distinción entre el agotado [épuisé] y la noción de fatiga o cansancio. Quien está cansado no 'dispone de ninguna posibilidad (subjetiva): no puede por ende realizar la más mínima posibilidad (objetiva)'. Es decir, quien está cansado, fatigado, no puede ya efectuar ninguna posibilidad. Desde este punto de vista quien está cansado agota la realización, ya no hace nada. Mientras que, el agotado agota todo lo posible, es decir, el agotamiento se ejerce sobre la posibilidad misma. No es que el agotado no pueda actuar, realizar, sino que el agotado no puede viabilizar ninguna posibilidad. [...] El horizonte del agotado deja de ser el de la posibilidad, su horizonte es sólo el de lo imposible”, en Guadalupe Lucero, "Agotar la lengua: Beckett a través de Deleuze", Daimon. Revista Internacional de Filosofía, 55 (2012): 131.

15. Colin Gardner, "How to Build a Desiring Machine: Quad I \& II (198I)", en Colin Gardner, Beckett, Deleuze and the Televisual Event: Peephole Art (Santa Barbara: University of California, 20I2), I54-I68. 
movimiento en continuo fluir. Valga decir que este tipo de imagen se corresponde, precisamente, con la imagen pura de inspiración bergsoniana, pues no en vano la filosofía de Deleuze adeuda un préstamo importante al pensamiento de Bergson, tal y como se constata en Le Bergsonisme (1966) y Proust et les signes (1964).

Para estudiar la especificidad del gesto repetitivo en Quad, y continuando con Deleuze, se desemboca en Différence et Répétition (1968), donde se hallan las consecuencias ontológicas de la repetición de lo diferente. El planteamiento de Deleuze en este libro coincide con el de Bergson, e incluso con el del propio Beckett, al rechazar las ataduras del hábito, pues éste establece cierta forma de monotonía existencial. En palabras de Beckett: "El hábito es el lastre que encadena el perro a su vómito." ${ }^{16}$ Un convencimiento que se nutre directamente de Proust y de su combinación de "tiempo", "memoria" y "costumbre". Así pues, la repetición beckettiana nada tiene que ver con el hábito. Es más bien un ritornelo, y como tal, repite lo distinto, justo lo que ocurre en Quad. Por eso, tampoco debe comprenderse como la repetición freudiana de "Más allá del principio del placer" (I920), basada en la reiteración de lo mismo ("Fort da"). Consiste, por otra parte, en un instrumento de alienación, que sirve asimismo como recurso de efecto cómico, característico en la obra de Beckett, presente sobre todo en obras como Esperando a Godot. ${ }^{17}$ Justamente decía Deleuze: "La repetición pertenece al humor y la ironía; es por naturaleza transgresión, excepción, manifestación constante de lo singular contra lo particular sometido a la ley". ${ }^{8}$

Esta dinámica de repetición centrífuga-centrípeta de Quad traza una visión espacial concreta, susceptible de ser estudiada a partir del concepto de tetradimensionalidad. Formulado hacia 1908 por Hermann Minkowski — quien fuera profesor de Einstein-, la principal novedad de dicho concepto radica en considerar el tiempo como la cuarta dimensión, y se suma por tanto a las tres dimensiones espaciales existentes. Ahora bien, puesto que esta teoría resulta incomprensible en virtud de la información que recibimos a través de los sentidos, en ocasiones se ha considerado una abstracción matemática imposible

I6. Samuel Beckett, Proust y otros ensayos, trad. Marcela Fuentealba (Santiago de Chile: Universidad Diego Portales, 2008), 54.

17. Nursel Içöz, "Repetition and Difference in Beckett's Works", en Marius Buning y Lois Oppenheim, eds., Beckett in the 19oos, Samuel Beckett Today/Aujourd'hui (Ámsterdam y Atlanta: Rodopi, I993), 28I-288.

I8. Gilles Deleuze, Diferencia y repetición, trad. Alberto Cardín (Madrid y Barcelona: Júcar, I988), 43. 
de aplicar a la realidad natural. ${ }^{19}$ Sin embargo, y contradiciendo la tradición científica, Minkowski sugirió que el espacio no es realmente como lo percibimos por los sentidos. Él abogaba por un espacio tetradimensional, que superase el espacio tridimensional descrito por la geometría clásica de Euclides (espacio euclideo tridimensional). Dicho de otra forma:

Hermann Minkowski comprendió que el espacio que percibían los sentidos era solamente una visión externa, una forma de manifestarse algunas de las propiedades geométricas del espacio universal real. ${ }^{20}$

Así pues, dado que los estímulos sensoriales no nos permiten captar el espacio como tetradimensional, la única forma de percibirlo es como puntos materiales manifestados en la realidad tridimensional. Por así decirlo, el espacio

19. "El propio Einstein no se sintió al principio demasiado seducido con la idea del espaciotiempo unificado, despreciando la nueva geometría tetradimensional de Minkowski como una pedantería 'superflua', pero volvió a la idea a su debido tiempo. La verdadera significación de este espacio-tiempo tetradimensional unificado es que posee una geometría común que mezcla completamente los fragmentos de espacio y los de tiempo", en Paul Davies, Sobre el tiempo: la revolución inacabada de Einstein (Barcelona: Crítica, 1996), 76. A pesar de su imposibilidad aparente, el respaldo a la teoría tetradimensional se deja sentir también con fuerza en el ámbito de las artes, como ocurre en el caso del cineasta Serguéi Eisenstein, quien influido por la idea de la cuarta dimensión, insta a considerar la validez de las propuestas de Minkowski: "Está comprobado que la connotación visual es en realidad una pieza, un elemento de... ¡una cuarta dimensión! En un espacio tridimensional, espacialmente inexpresivo, y sólo emergiendo y existiendo en la cuarta dimensión (el tiempo añadido a las tres dimensiones). ¿La cuarta dimensión? ¿Einstein? ¿O misticismo? ¿O un chiste? Es el momento de dejar de tenerle miedo a este nuevo conocimiento de una cuarta dimensión. El propio Einstein nos lo asegura: 'Se apodera del no matemático un misterioso temblor cuando escucha acerca de cosas "tetra-dimensionales", un sentimiento parecido a los que despiertan los pensamientos de lo oculto. Y sin embargo no hay mayor evidencia que aquella de que el mundo en el que vivimos es un continuum de espacio-tiempo tetra-dimensional'." ("The visual overtone is proved to be an actual piece, an actual element of — a fourth dimension! In three-dimensional space, spatially inexpressible, and only emerging and existing in the fourth dimension (time added to the three dimensions). The fourth dimension? Einstein? Or mysticism? Or a joke? It's time to stop being frightened of this new knowledge of a fourth dimension. Einstein himself assures us: 'The nonmathematician is seized by a mysterious shuddering when he hears of 'four-dimensional' things, by a feeling not unlike that awakened by thoughts of the occult. And yet there is no more common-place statement than that the world in which we live is a four-dimensional space-time continuum'", en Serguéi Eisenstein, Film Form. Essays in Film Theory, and The Film Sense [Cleveland y Nueva York: Meridian Books, 1957], 69-70) (trad. de la autora).

20. A. A. Sazânov, El universo tetradimensional de Minkowski (Moscú: Editorial Mir, I990), I4. 
tetradimensional se halla sumergido en el espacio euclídeo tridimensional. De un modo gráfico, la representación básica de este tipo de espacio se plasma en lo que Minkowski denomina "plano pseudoeuclídeo". Se trata de un espacio bidimensional con propiedades métricas pseudoeuclídeas. ${ }^{2 \mathrm{I}}$ Desde esta perspectiva, la superficie bidimensional del tablero de Quad se antoja un ejemplo inmejorable para comprender el espacio tetradimensional, ya que las propiedades lineales del plano pseudoeuclídeo coinciden con las del plano euclídeo mismo. ${ }^{22}$ Además, según apunta Minkowski, el plano pseudoeuclídeo está atravesado por lineas isótropas, o vectores isótropos: rectas que dividen el espacio en cuatro sectores. La descripción parece encajar nuevamente con el espacio de Quad, por estar organizado en cuatro frentes: los cuatro lados del cuadrado, recorridos por cuatro trayectos lineales iguales pero distintos.

Dicho esto, téngase en cuenta que los cuatro sectores isótropos no sólo dividen el espacio, sino también el tiempo, ya que a partir de Minkowski el tiempo y el espacio no pueden pensarse por separado. Y así, en función del sector, los individuos se situarán en el futuro o en el pasado absolutos. ${ }^{23}$ Elevado a una representación gráfica tridimensional, este espacio adopta un aspecto parecido al de un reloj de arena: un doble cono unido por un plano ortogonal, llamado hipercono isótropo, considerado un conjunto tridimensional de puntos en un espacio tetradimensional (fig. 2). Gracias a este novedoso planteamiento surge, por vez primera, una explicación sintética de la naturaleza del tiempo y del espacio; cuestión que tanto preocupaba a Samuel Beckett, y que él mismo intenta resolver por medio de obras tan elaboradas, aunque aparentemente sencillas, como Quad, en la que convoca una personalísima reunión de elementos temporales y espaciales.

2I. "Cuando miramos un dibujo plano de objetos tridimensionales, la experiencia multifacética de las percepciones sensoriales, de un modo casi insensible para nuestra conciencia, hace el desciframiento, poniendo en lugar de las imágenes convencionales las representaciones correctas de los objetos. Pero como las relaciones métricas [p]seudoeuclídeas nunca han sido percibidas por nuestros órganos sensoriales, la imagen convencional de esas relaciones no sólo no le recuerdan nada a nuestra conciencia, sino que contradicen todas las representaciones geométricas a que estamos acostumbrados", en Sazânov, El universo tetradimensional, iı8.

22. Jacques Bros y Ugo Moschella, "The Geometry of Relativistic Spacetime: from Euclid's Geometry to Minkowski's Spacetime”, en Thibault Damour, Olivier Darrigol y Vincent Rivasseau, eds., Einstein, 1905-2005: Poincaré Seminar (Basel, Boston y Berlín: Birkhäuser Verlag, 2006), 59-133.

23. Gregory L. Naber, The Geometry of Minkowski Spacetime: An Introduction to the Mathematics of the Special Theory of Relativity (Mineloa y Nueva York: Dover Publications, 1992), I8. 
2. Hipercono isótropo de Hermann Minkowski

(ilustración de la autora).

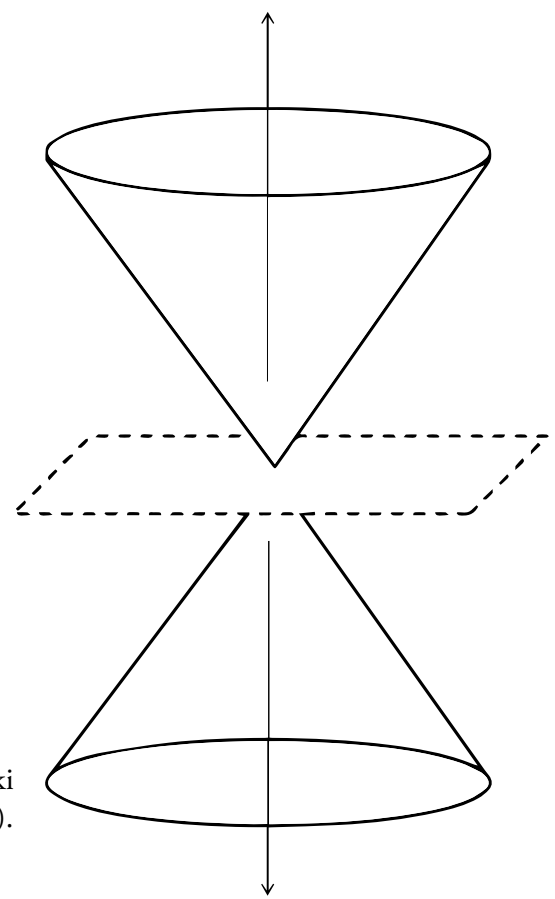

Giacometti a través del tiempo

Agotadores, los recuerdos. Por eso no hay que pensar en ciertas cosas, cosas que te importan, o mejor sí, hay que pensar en ellas, porque si no pensamos en ellas corremos el riesgo de encontrarlas, en la memoria, poco a poco. ${ }^{24}$

Como se ha visto, lejos de considerarse entidades separadas, las nociones de tiempo y espacio en el universo tetradimensional se estiman dos variables íntimamente ligadas, que componen un concepto unificado de espacio-tiempo, esto es, un continuo espacio-temporal. En este punto, la teoría de Hermann

24. Samuel Beckett, Relatos, trad. Félix de Azúa (Barcelona: Tusquets, 1997), 31. 
Minkowski casa a la perfección con la teoría de la relatividad elaborada por su discípulo, Albert Einstein. ${ }^{25}$ No así con la de Henri Bergson, quien sostiene la necesidad de diferenciar el espacio y el tiempo. Bergson llega incluso a contradecir abiertamente a Einstein en el libro Durée et Simultanéité (I922); un asunto que derivó en malentendido y tuvo considerable repercusión en la opinión pública, y dio pie a una sonada polémica — lo que llevó a Bergson a retractarse y matizar su postura. Ocurre que el tiempo de Bergson no es el tiempo que estudia las leyes de la física, sino el tiempo como dimensión ontológica, lo que él llama tiempo puro. Así entendido, el tiempo es la conjunción de duración, devenir e intensidad; no un fenómeno matemáticamente mensurable. ${ }^{26}$

Así pues, mientras que el hipercono isótropo de Minkowski representaba el espacio y el tiempo unidos, Bergson plasmará el tiempo en un diagrama distinto: un único cono en cuyo interior se genera un flujo cíclico constituido por diversos planos de hechos psicológicos, emocionales, cognitivos; en definitiva, eventos ontológicos, perceptivos y de memoria (fig. 3). De esta duración pura bergsoniana deriva, por tanto, la idea de una perenne construcción del "yo" individual mediante el funcionamiento continuo del cono, que actualiza el pasado en el presente. ${ }^{27}$ Por el contrario, en la teoría de Minkowski, el "yo" surge en un momento específico del presente, localizado en la intersección que unía los dos

25. "Es justo el principio de la relatividad de Einstein. El tiempo no es una línea que ocurre por sí misma, independiente del espacio, sino que es una línea cuya métrica está esencialmente afectada por el espacio y por el movimiento. Entonces el tiempo es una dimensión (algo referente a la medida o mensura) que no sería independiente de las tres dimensiones espaciales, sino que constituiría, junto con ellas, una cuarta dimensión del mundo. Para su representación gráfica podría servir el cronótropo de Minkowski [...]”, en Zubiri, Espacio. Tiempo. Materia, 230.

26. "La duración es el nombre de una serie fluida, en constante movimiento y de dirección irreversible. Es, por definición, la negación del estaticismo o de un orden mecánico en el que hay 'antes' y 'después'. Es el ámbito reservado y lleno para la pura fluidez, para el puro proceso. Bergson utiliza, en su descripción de este rasgo de la duración múltiples ejemplos, que se encuentran cortados por un denominador común: la referencia a los estados de conciencia y la referencia al tiempo. [...] el tiempo y, sobre todo, la conciencia personal son la misma duración, son confirmaciones —es devenir temporal y en actividad de sujeto— de los rasgos de la propia duración", en Izuzquiza Otero, Henri Bergson, 36-37.

27. "Refiriendo la duración a los estados de conciencia, afirma Bergson que: la duración pura es la forma que toma la sucesión de nuestros estados de conciencia cuando nuestro yo se deja vivir, cuando se abstiene de establecer una separación entre el estado presente y los estados anteriores." O también: “(los estados que pretendemos definir de nuestra vida psicológica...) puede decirse que no son elementos distintos. Se siguen los unos a los otros en un 'transcurso sin fin”", en Izuzquiza Otero, Henri Bergson, 37. 
3. Cono temporal de Bergson (ilustración de la autora).

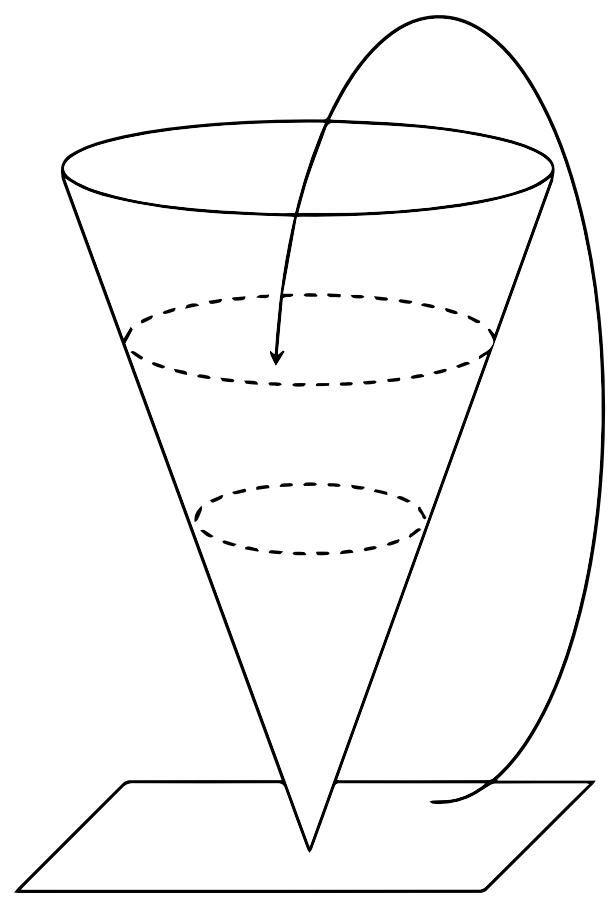

conos espacio-temporales, uno dirigido hacia el pasado y el otro hacia el futuro:

Obsérvese que el "futuro" y el "pasado" — como las mitades superior e inferior de un reloj de arena — son dos regiones separadas cuyo único punto en común es nuestro “aquí-y-ahora”. El "presente” rodea esos dos conos y es una región única conectada. ${ }^{28}$

En el intento de sintetizar ambas posturas — la de Minkowski y la de Bergson- respecto al tiempo, acudimos al texto de Alberto Giacometti, "Le rêve, le Sphinx et la mort de T.”, escrito en 1946. En sus páginas se materializa un amplio catálogo de saltos temporales, que combinan los recuerdos biográficos del autor con vivencias especialmente impactantes, sueños, fabulaciones y estados emocionales que transitan la ansiedad y el terror. De esta forma, el texto permite acceder a la experiencia ontológica de Giacometti a través de la percepción de un continuo temporal, integrado por distintos eventos psicológicos.

28. John Richard Gott, Los viajes en el tiempo y el universo de Einstein, trad. Luis Enrique de Juan (Barcelona: Tusquets, 2003), 76. 
Esto enlaza directamente con las ideas de durée y de tiempo puro de Bergson.

Ahora bien, el objetivo de Giacometti al escribir este relato no es otro que poner en orden semejante mezcolanza de sucesos, y con dicho fin diseñará su propio diagrama espacio-tiempo. El resultado es un plano circular en forma de disco, en el que el artista fija sus recuerdos y estados de ánimo como puntos en el tiempo, a los cuales dota, además, de una localización en el espacio. ${ }^{29}$ Así pues, mediante una figura geométrica, Giacometti obtiene una visión global, dinámica pero organizada, de sus vivencias más señaladas, y lo hace desde una perspectiva temporal, pero también espacial. Logra de este modo vehicular su propia concepción espacio-tiempo, integrándola en un todo coherente. Es por eso que, al unir las dimensiones espaciales con la temporal, el disco de Giacometti alcanza un resultado parangonable al hipercono de Minkowski. En este sentido, podemos afirmar que este texto de Giacometti — junto con el diagrama que lo acompaña- sirve como instrumento a medio camino entre las posturas de Bergson y Minkowski.

El texto en cuestión comienza con un sueño en el que Giacometti contempla aterrorizado una monstruosa arańa que descansa a los pies de su cama, acariciada por una mano femenina. Se deduce del propio relato que esta visión onírica encarna la inseguridad del artista frente al encuentro sexual con la mujer y el trauma del riesgo sifilítico. Movido por semejante temor, Giacometti golpea al insecto con un largo bastón hasta matarlo. No resulta complicado inferir aquí una lectura edípica. En cualquier caso, el miedo a las arañas resulta común entre los surrealistas, siendo muy conocido el caso de Luis Buñuel, con quien Giacometti mantenía además una buena amistad. Al continuar el texto, Giacometti indica que el sueño se relaciona con el hecho de estar esperando los síntomas de una enfermedad venérea. En este punto, la historia experimenta un salto atrás en el tiempo: el artista afirma que permanece atento a la aparición de la enfermedad desde el día en que tuvo noticia del cierre del Sphinx, un conocido prostíbulo parisino, que Giacometti apreciaba especialmente (resulta interesante apuntar que no sólo Giacometti era asiduo del Sphinx, sino que también lo era Samuel Beckett, quien solía acompañar a su amigo escultor). En palabras de Giacometti:

29. Sobre esta rueda espacio-temporal véase Georges Didi-Huberman, Le cube et le visage. Autour d'une sculpture d'Alberto Giacometti (París: Éditions Macula, 1993). 
Yo esperaba aquella enfermedad desde el sábado anterior, en que, habiéndome enterado, hacia las seis de la tarde, de que iban a cerrar para siempre el Sphinx, corrí hacia allí, pues encontraba intolerable la idea de no volver a ver aquella sala donde había pasado tantas horas, tantas veladas desde su apertura, y que era para mí un lugar maravilloso antes que nada. ${ }^{30}$

A continuación, el relato sigue viajando hacia atrás en el tiempo: el artista se enteró del cierre del burdel en una comida con amigos en la que Albert Skira, antiguo editor de la revista Minotaure, propuso a Giacometti escribir un texto sobre la muerte de T., con idea de publicarlo en su nueva revista, Labyrinthe. ${ }^{31}$ Así pues, vemos cómo el sueño de la araña desencadena un meandro memorístico que permite a Giacometti volver a este episodio de su pasado, del cual regresa finalmente al presente para narrar la muerte de T. Entre otras cosas, Giacometti cuenta cómo arregla el cadáver, la impresión insignificante del cuerpo, los primeros signos de putrefacción, y sobre todo, una extraña sensación que experimenta ante el rostro de aquella figura sin vida, que lleva al artista a realizar un nuevo salto temporal. Éste lo posiciona ahora ante el recuerdo de sus recientes problemas de percepción:

En sentido inverso, acababa de experimentar lo que había sentido algunos meses atrás ante los seres vivos. En aquel momento, empezaba a ver las cabezas en el vacío, en el espacio que las rodea. [...] Ya no era una cabeza viva, sino un objeto que yo miraba como a cualquier otro objeto [...] como algo vivo y muerto a la vez. [...] Todos los vivos estaban muertos, y esta visión se repetía a menudo, en el metro, en la calle, en el restaurante, con mis amigos. [...] Pero al mismo tiempo que los hombres, los objetos sufrían una transformación, las mesas, las sillas, las ropas, la calle, hasta los árboles y los paisajes. ${ }^{32}$

Una vez concluido el pasaje de la muerte de T., se produce un salto temporal hacia delante, que sitúa a Giacometti "algunos días después de escribir de un golpe lo que acabamos de leer", sentado en un café parisino, revisando el texto

30. Alberto Giacometti, Escritos, trad. José Luis Sánchez Silva (Madrid: Síntesis, 200I), 63-64. 3I. “'Cuando Alberto Giacometti escribió 'El sueño, el Sphinx y la muerte de T.' (el cadáver era el de Tonio Pototsching, administrador del complejo de talleres de la Rue Hippolyte-Maindron) eran los años en que estaba 'racionalizando' su experiencia personal con el espacio", en María García Yelo, "El sublime espacio de Alberto Giacometti", Anales de Historia del Arte, núm. I4 (2004): 239.

32. Giacometti, Escritos, 65-66. 
e intentando reescribir el sueño de la araña de otra manera, pues empieza a darse cuenta de las distintas trayectorias temporales que atraviesan su historia, la cual aparece configurada sucesivamente como un relato dentro de otro. De este modo, Giacometti vislumbra el nudo gordiano de la problemática que late en su texto. Finalmente, se hace consciente de la intersección del tiempo psicológico (Bergson) y del tiempo cronológico (Minkowski):

Había una contradicción entre la manera afectiva de reflejar lo que me alucinaba y la secuencia de hechos que quería contar. Me encontraba ante una masa confusa de tiempo, acontecimientos, lugares y sensaciones. Trataba de encontrar una posible solución. [...] El tiempo en mi historia iba a contracorriente, del presente al pasado, pero con retornos y ramificaciones. ${ }^{33}$

Es por eso que Giacometti intenta reorganizar su escrito siguiendo un esquema que permita narrar ordenadamente la experiencia de sus estados anímicos y su propia percepción del tiempo, combinando pasado, presente, ensońaciones, recuerdos y sentimientos. He aquí que, como broche final del artículo, Giacometti inventa su diagrama espacio-temporal: un disco que permite situar los distintos hechos de manera simultánea, aun teniendo una localización distinta en el continuo fluir espacio-temporal. El artista explica la invención de dicho diagrama como una solución que se le presenta de manera clara y evidente para intentar resolver el enredo de vectores espacio-temporales:

De pronto, tuve la sensación de que todos los acontecimientos se producían simultáneamente a mi alrededor. El tiempo se hacía horizontal y circular, era espacio al mismo tiempo, e intenté dibujarlo. [...] Con un extraño placer, me veía a mí mismo paseando por el disco tiempo-espacio, y leyendo la historia erigida ante mí. La libertad de comenzar por donde quisiera, por ejemplo por el sueńo de 1946, para concluir, después de dar toda la vuelta, algunos meses antes, ante los objetos [...]. Me interesaba mucho la orientación de cada hecho en el disco. ${ }^{34}$

No se pase por alto el componente espacial que Giacometti identifica en este diseño: un disco de naturaleza temporal en el que, sin embargo, el propio artista puede situarse y recorrerlo a su antojo, saltando del presente al pasado

33. Giacometti, Escritos, 67-68.

34. Giacometti, Escritos, 69-70. 
y viceversa. Desde este punto de vista cabe apuntar que el tiempo en Giacometti se asemeja bastante al tiempo imaginario acunado por Stephen Hawking. ${ }^{35}$ Por este vocablo se entiende una noción de tiempo que va más allá del tiempo natural que experimentamos de manera unidireccional. Consiste, por tanto, en un modelo temporal capaz de moverse sin ataduras, libre de la percepción lineal que avanza únicamente desde el pasado hacia el futuro. De hecho, es sabido que el tiempo imaginario es otro concepto utilizado para referirse al tiempo tetradimensional. No en vano, decíamos que Giacometti experimenta una percepción del tiempo muy parecida a la de Minkowski. Es más, la conexión con el universo tetradimensional se hace todavía más llamativa si se tienen en cuenta los paralelismos del texto de Giacometti con la teorización de Minkowski acerca del conjunto temporal "pasado-presente-futuro".

Partimos del hecho de que el modelo clásico del universo utiliza un criterio analítico tridimensional, según el cual sólo existe el presente, ya que el pasado propiamente no existe (ha pasado) y el futuro tampoco (aún no ha ocurrido). Frente a esta postura, Minkowski contrapone una visión del universo en la que todos los objetos materiales participan de manera conjunta en un proceso continuo de transcurso del tiempo, fusionando los tres registros temporales tradicionalmente compartimentados. La base de esta teoría estriba en entender la realidad como un proceso de revelación o manifestación, en el cual conviven los sucesos pasados - ya revelados - y también los futuros — que todavía permanecen latentes. Todos ellos ocupan su lugar en la linea del universo, esto es, la trayectoria gráfica del movimiento que experimenta cada punto material (puntos del universo) en el plano pseudoeuclídeo.

Ocurre que por causas de limitación sensorial no podemos percibir visualmente una línea del universo en su extensión real, sino que en cada instante percibimos sólo puntos aislados de la misma, los puntos del universo, en forma de puntos materiales. Pero esto no indica necesariamente que sólo exista este presente de cada punto del universo, sino que en realidad coexiste con el resto de puntos que componen su trayectoria (línea del universo). Así pues, cada punto del universo es simultáneo a los puntos pasados de su línea de universo, los cuales se consideran objeto material ya formado, revelado. En este sentido, la diferencia entre pasado y futuro será el límite entre lo manifiesto y lo no

35. Stephen Hawking y Roger Penrose, La naturaleza del espacio y el tiempo, trad. Javier García Sanz (Madrid: Debate, 1996). 
DOI: http://dx.doi.org/10.22201/iie.18703062e.2017.1.2590

66 MARÍA DEL CARMEN MOLINA BAREA

manifiesto. De forma que el presente del individuo se corresponderá con el instante actual del acontecimiento de manifestación. En palabras de Minkowski:

Las líneas de universo se encuentran en proceso de desarrollo. Existen ya, se han manifestado ya, en aquellas de sus partes que se refieren al pasado, y todavía no existen, no se han revelado en la parte del futuro. El límite de la parte ya revelada de la línea de universo es el límite entre el pasado y el futuro, es el mismo "instante actual" que continuamente pasa del pretérito al futuro. De este modo vemos en el proceso de transcurso del tiempo, que abarca a todo el mundo material, un proceso de crecimiento, de prolongación, de revelación de las líneas de universo o proceso de revelación o manifestación universal. ${ }^{36}$

En este extremo, cabe argumentar una equivalencia directa entre la realidad tetradimensional de las líneas de universo de Minkowski y el diagrama espacio-temporal de Giacometti, en tanto que ambos planteamientos sugieren una coexistencia entre niveles pasados, presentes y futuros. Dicho sistema de revelación permitiría incluso, en teoría, viajar en el tiempo hacia el pasado y conocer nuestros "yoes" pretéritos, los cuales, aunque pasados, siguen existiendo. Ello sería posible, claro está, de no encontrarnos limitados humanamente a efectos de percepción sensorial. Y es que en nuestras circunstancias, sólo podemos percibir las líneas isótropas que pasan por nuestro punto del universo. ${ }^{37}$ No obstante, vemos cómo Giacometti logra superar esta restricción por medio de su escritura, a lo largo del texto sobre el Sphinx, en el que consigue efectivamente viajar hacia puntos pasados de su línea del universo y contemplar sus otros "yoes".

En cualquier caso, en Giacometti parecen subsistir también no pocas resonancias bergsonianas. ${ }^{38}$ Téngase en cuenta, sin ir más lejos, que el concepto

36. Sazânov, El universo tetradimensional, $2 \mathrm{II}$.

37. Para Minkovski "existe el pasado, pero no en el sentido del 'criterio tridimensional de la existencia', no en el 'instante actual', sino en el sentido del 'criterio cuatridimensional de la existencia': existe lo que tiene lugar, se ha manifestado, se ha materializado en el espacio [p] seudoeuclídeo universal. Las percepciones visuales nos descubren lo que en el mundo existe en realidad, pero no todo, sino sólo lo que se encuentra en las líneas isótropas que pasan por nuestro punto del universo. En esas líneas isótropas no hay ni un solo punto de universo pasado de nuestra línea de universo propia y, por eso, no vemos nuestros estados pretéritos", en Sazânov, El universo tetradimensional, 236.

38. Manfred Milz, "Echoes of Bergsonian Vitalism in Samuel Beckett's early Works", en Minako Okamuro, Naoya Mori, Bruno Clément, Sjef Houppermans, Angela Moorjani y Anthony 
de durée aporta una idea de tiempo "no cronológico", lo que implica la ruptura de la unidireccionalidad temporal, para implantar en su lugar la coexistencia de pasado y presente. ${ }^{39}$ Diríamos entonces que el tiempo en Bergson se desvincula por completo de la sucesión cronológica, para adecuarse, más bien, a una simultaneidad psicológica. Todo ello viene dado por el continuo fluir de la duración pura, que rompe los márgenes tradicionales del "antes" y el "después". Tomando prestada la expresión de Paolo Virno, hablaríamos, pues, de un "procedimiento contratemporal". ${ }^{\circ}$ Gilles Deleuze ha sabido apreciar especialmente esta convivencia del espectro temporal en la teoría de Bergson:

El pasado y el presente no designan dos momentos sucesivos, sino dos elementos que coexisten: uno, que es el presente que no cesa de pasar; el otro, que es el pasado y que no cesa de ser, pero mediante el cual todos los presentes pasan. ${ }^{4 r}$

Asimismo, en la redacción de su artículo, Giacometti juega con la síntesis de pasado y presente en una constante confusión de realidad diurna y realidad onírica. A este respecto, sirva mencionar que el tiempo bergsoniano encuentra su mejor materialización en la mente inconsciente y en el onirismo, o lo que es lo mismo, en la imbricación de sueño y vigilia. Pues precisamente, los sucesos que se desarrollan en los sueños manifiestan con frecuencia una alteración notoria del orden cronológico habitual. ${ }^{42}$ Igualmente, fenómenos oníri-

Uhlmann, eds. Borderless Beckett/Becket Sans Frontières, Samuel Beckett Today/Aujourd'hui I9 (Ámsterdam y Nueva York: Rodopi, 2008), I43-I56.

39. "La aplicación de la duración al tiempo obliga a reformular las bases mismas del proceso temporal habitual: la distinción entre pasado, presente y porvenir, la consideración del instante, la especificidad propia — no ya la distinción — de pasado, presente y porvenir. En definitiva, una reestructuración total del concepto de tiempo que se encuentra dominado por las categorías del espacio. Se trata de una operación purificadora, catárquica del concepto de tiempo, que Bergson quiere recuperar totalmente", en Izuzquiza Otero, Henri Bergson, 43.

40. Paolo Virno, El recuerdo del presente. Ensayo sobre el tiempo histórico, trad. Eduardo Sadier (Barcelona: Paidós, 2003), 35 .

4I. Gilles Deleuze, El bergsonismo, trad. Luis Ferrero (Madrid: Cátedra, 1996), 59.

42. 'Como todos los sueños convincentemente vívidos, el 'sueño' de Giacometti presenta múltiples capas. La resplandeciente superficie surrealista de impresiones recolectadas flota sobre recuerdos re-experimentados, hilvanados hacia atrás y hacia delante en el tiempo y que en última instancia conducen al descubrimiento por parte del artista de un modo formal de contar la historia de su vida. Temas y formas se tejen y entretejen en cuatro dimensiones: horizontalidad, verticalidad, tiempo, y espacio. Eventos externos son interiorizados y recreados por el sońante como impresionantes y terroríficas obras de arte." ("Like all compellingly vivid dreams, Giacometti's 
cos similares, como el sonambulismo y el automatismo mental, ponen también al individuo en sintonía con la experiencia del tiempo puro. He aquí que los artistas del surrealismo se hacen eco de esta influencia, por estar muy relacionada con los principales objetivos del grupo: la libertad del inconsciente, del sueño, de las pulsiones instintivas y de las creaciones artísticas surgidas al margen del control racional. Por ello no es extraño que Giacometti se viera inserto en esta revalorización de la temporalidad de los sueños, cuyo protagonismo refleja magistralmente en el texto para Labyrinthe.

El sueńo nos coloca precisamente en estas condiciones, porque el sueńo, al reducir el juego de las funciones orgánicas, modifica especialmente la superficie de comunicación entre el yo y las cosas exteriores. Entonces no medimos la duración, pero la sentimos; de cantidad pasa al estado de calidad; la apreciación matemática del tiempo transcurrido deja de hacerse, cediendo el puesto a un instinto confuso, capaz, como todos los instintos, de cometer groseros desprecios y también a veces de proceder con una seguridad extraordinaria. 43

De este modo, la temporalidad de "El sueño, el Sphinx y la muerte de T." procede como en un sueńo, es decir, como la percepción dislocada del tiempo que experimentamos al soñar. Es más, se articula como el producto de un sueño dentro de otro sueño, en la medida en que Giacometti consigue enlazar varios estados oníricos, sin saber exactamente cuándo termina uno y empieza otro. Casi a la manera kafkiana, ni siquiera el propio autor es capaz de distinguirlos: 'Me desperté en ese momento, pero me desperté en el sueño, que continuaba." 44 O como reza el poema de Beckett: "Sueño/sin fin/ni tregua/ alguna." 45 Curiosamente, este fenómeno se da también en la obra de varios artistas surrealistas, siendo muy conocido el caso de Luis Buñuel. Véase por

dream is multilayered. The shimmering surrealist surface of recollected impressions floats above reexperienced memories weaving back and forth in time and ultimately leading to the artist's discovery of a formal way to tell his life story. Themes and forms are woven and interwoven in four dimensions - horizontality, verticality, time, and space. External events are internalized and re-created by the dreamer as awe-inspiring and terrifying works of art", en Laurie Wilson, Alberto Giacometti: Myth, Magic, and the Man [New Haven, Londres: Yale University Press, 2003], 2II) (trad. de la autora).

43. Henri Bergson, Memoria y vida. Textos escogidos por Gilles Deleuze, trad. Mauro Armiño (Madrid: Alianza, 1987), Io.

44. Giacometti, Escritos, 62.

45. Samuel Beckett, Quiebros y poemas, trad. Loreto Casado (Madrid: Árdora, 2005), 5 I. 
ejemplo la película Le charme discret de la bourgeoisie (El discreto encanto de la burguesía, 1972), basada en una combinación interminable de sueńos concatenados. El resultado de este filme es muy similar a los efectos logrados en el artículo de Giacometti: ni en la película ni en el texto se alcanza una idea clara de un punto fijo en el tiempo a partir del cual identificar un pasado o un presente, pues todo se halla inmerso en una neblina que confunde la posición espacio-temporal con el sueño y la vigilia. Precisamente en esto radican, según Bergson, las cualidades perceptivas del tiempo puro.

Por otro lado, el cine buñueliano está plagado de continuos guiños al flashback y flashforward, recurso que alcanza su máxima aplicación en El ángel exterminador (1962). A lo largo del filme se repiten varias escenas que, aun siendo las mismas, difieren en cada repetición al introducirse una irracional coexistencia de temporalidades. En esta línea, Deleuze ha declarado que Buñuel es el creador cinematográfico de la imagen-tiempo, una tipología de imagen que nace justamente a partir de la repetición de lo diferente, que quiebra la coherencia espacio-temporal. ${ }^{4}{ }^{6}$ Esto entronca con la imagen pura bergsoniana, cuyo referente directo se vio en Quad: una imagen surgida de la repetición de lo diferente (ritornelo). No es tampoco coincidencia que Deleuze considere la imagen-tiempo una variante de la imagen-sueño, puesto que, al igual que ésta, posee la capacidad de alterar la disposición lineal del tiempo y del espacio. Este cruce de imagen-tiempo e imagen-sueño se aprecia desde la primera película de Buñuel hasta sus últimos filmes: desde los intertítulos de Un chien andalou (Un perro andaluz, I929), que sorprenden por su inadecuación a una supuesta discursividad argumental, hasta los peregrinos de La voie lactée (La Vía Láctea, 1969), que se transportan a través del tiempo y del espacio, saltando entre distintos lugares y épocas históricas. Finalmente, ello es posible gracias al solapamiento repetitivo de recuerdos, imaginación y ensueño. Incluso Samuel Beckett expone dicho fenómeno en términos semejantes:

La identificación de la experiencia inmediata con la pasada, la repetición de una acción o reacción pasada en el presente, produce en efecto una mezcla entre lo ideal y lo real, imaginación y aprehensión directa, símbolo y sustancia. 47

En este punto, huelga decir que Buñuel es otro surrealista que se mueve en la órbita de Bergson. Pudo recibir su influencia en el contexto de la Residencia de

46. Gilles Deleuze, La imagen-movimiento. Estudios sobre cine I, trad. Irene Agoff (Barcelona: Paidós, 1984), I86.

47. Beckett, Prousty otros ensayos, 86. 
Estudiantes de Madrid, donde el joven cineasta se alojó entre I917 y 1925, y en cuyas instalaciones el propio Bergson impartió una conferencia en 1916. ${ }^{48}$ Asimismo, merece la pena recordar que Buñuel realizó su incursión como aprendiz en el mundo del cine de manos de Jean Epstein, a quien Paul Hammon llamó precisamente "teosofista bergsoniano". De ahí, pues, el interés de Buñuel en el tratamiento del espacio-tiempo:

[Buñuel] ya había exhibido su desprecio por la linealidad cronológica canónica en su temprano cuento de 1923 titulado Por qué no uso reloj, en el que citó a Einstein y jugueteó desenfadadamente con el relativismo del vector temporal. Y, más tarde, la versatilidad temporal fue expresada por Dalí en sus famosos "relojes blandos", que pintó desde 1930.49

Pues bien, del mismo modo que Buñuel moldea una percepción desarticulada del tiempo, fundamentada en la repetición de lo diferente, así también procede Giacometti ante el cadáver de T., cuando afirma experimentar una sensación equivalente a aquella que había experimentado hacía meses frente a otros objetos y personas. Ocurre igualmente con la reminiscencia de la enfermedad venérea, suscitada por el cierre del Sphinx, y en general, con el gesto mismo de actualizar (traer al momento actual) sus impresiones afectivas al rememorar dichos sucesos, en un alarde similar al de Proust y su famosa magdalena. Así pues, lo vivido por Giacometti no es simplemente un déjà vu, sino más bien, una situación pasada que se desarrolla en el presente; por tanto, un evento que cada vez que se repite es el mismo pero también diferente. ${ }^{50} \mathrm{Al}$

48. En este contexto se localiza asimismo la influencia de Ortega y Gasset, cuya figura se encuentra íntimamente relacionada con el ambiente cultural de la Residencia de Estudiantes. Es muy significativo que en ${ }^{1923}$, Ortega escribiese el prólogo del libro Geometrías no euclidianas de Roberto Bonola, que se publicó en la colección Biblioteca de Ideas del siglo xx de la editorial Calpe, creada y dirigida por el propio Ortega. Un año antes, en I922, había visto la luz en la misma editorial el libro Teoría de la relatividad de Einstein y sus fundamentos físicos, de Max Born. Todo ello pone de manifiesto el interés de Ortega por la teoría tetradimensional. No en vano, en ese mismo año de 1922, Einstein visitaba la Residencia de Estudiantes para dar una conferencia sobre la teoría de la relatividad.

49. Román Gubern, Proyector de luna. La generación del 27 y el cine (Barcelona: Anagrama, 1999), 4I4.

50. Aquí se aprecia nuevamente la influencia de Bergson respecto de la ilusión entretejida por la percepción, el recuerdo y la imagen: "Procede de que el recuerdo reavivado, consciente, nos da la impresión de ser la percepción misma que resucita bajo una forma más modesta, y nada más que 
seguir a Deleuze, tal procedimiento supondría una libre adaptación del "eterno retorno" de Nietzsche, metamorfoseado en el diagrama temporal de Bergson: el cono que acoge un fluido continuo de episodios psicológicos, los cuales se repiten de manera distinta. En este sentido, si el diseńo de Quad se ajustaba al ritornelo, el tiempo en Giacometti ejemplifica lo que podríamos denominar el "eterno retorno de lo diferente".

\section{Conclusiones: el espacio-tiempo de la esquizofrenia}

Desde el comienzo de mi crisis siempre he estado persuadido de ser un enfermo de tiempo. ${ }^{\text {II }}$

El estudio de la percepción espacio-temporal en Beckett y Giacometti desemboca, finalmente, en el ámbito de la patología mental. A este respecto, resulta llamativo comprobar que ambos autores acceden a la comprensión del espacio-tiempo por medio de procedimientos similares a los que emplea la mente esquizofrénica. De hecho, podríamos decir que los recursos desarrollados por Beckett y Giacometti coinciden en su mayoría con los síntomas que presenta el individuo esquizoide al percibir el tiempo y el espacio. Para comprobarlo, remitiremos a la obra del psiquiatra Eugène Minkowski (que comparte apellido, aunque ningún lazo familiar, con el padre de la teoría tetradimensional). Considerado el fundador de la fenomenología psiquiátrica, Eugène Minkowski hizo contacto con la esquizofrenia en la clínica Burghölzli de Zúrich, en la cual trabajó también el conocido psicoanalista C. G. Jung. Minkowski pronto sistematizó su propia concepción de la enfermedad, muy influenciado por Husserl, y especialmente por Bergson —una influencia que reconoció en repetidas

esta percepción. Entre la percepción y el recuerdo habría una diferencia de intensidad o de grado, pero no de naturaleza. [...] El recuerdo de una sensación es algo capaz de sugerir esta sensación, quiero decir, de hacerla renacer, débil al principio, más fuerte después, más fuerte paulatinamente a medida que la atención se fija más sobre ella. [...] En efecto, la sensación es esencialmente actualidad y presente; pero el recuerdo, que la sugiere desde el fondo del inconsciente de donde emerge a duras penas, se presenta con ese poder sui generis de sugestión que es la marca de lo que no es, de lo que todavía querría ser. Apenas ha tocado la imaginación la sugestión cuando la cosa sugerida se dibuja en estado naciente, y por ello resulta tan difícil distinguir entre una sensación débil que se experimenta y una sensación débil que se rememora sin fechar”, en Bergson, Memoria y vida, 50-5I.

5I. Eugène Minkowski, El tiempo vivido. Estudios fenomenológicos y psicológicos, trad. Ángel Sáiz Sáez (México: Fondo de Cultura Económica, 1973), 308. 
ocasiones..$^{52}$ Entre sus investigaciones destaca la trilogía La Schizophrénie (1927), Le Temps vécu (1933) y Traité de Psychopathologie (1966).

Para comprender en primer lugar la dimensión espacial beckettiana, mencionaremos la teoría de Minkowski acerca del pensamiento espacial de los esquizofrénicos, o lo que es lo mismo, el llamado racionalismo mórbido o geometrismo mórbido. Este principio patológico se manifiesta en una atrofia hiperbólica del planeamiento espacial, que lleva al enfermo a concebir el espacio en virtud de la geometría, del razonamiento lógico-matemático y de una particular "manía de simetría". Según el testimonio de Minkowski:

Veremos de esta manera cómo el esquizofrénico, privado de la facultad de asimilar todo lo que es movimiento y duración, tiende a construir su comportamiento con factores y con criterios cuyo dominio propio, en la vida normal, es únicamente la lógica y las matemáticas. ${ }^{53}$

Semejante actitud recuerda en sus síntomas al ordenamiento espacial desplegado por Beckett en Quad. Desde esta perspectiva, cabe afirmar que la tendencia obsesiva de Beckett hacia la geometrización se vincula directamente con el perfil etiológico del esquizofrénico estudiado por Minkowski —una equivalencia que Yann Mével ha subrayado. ${ }^{54}$ Tampoco resulta extraña esta similitud, teniendo en cuenta que el propio Minkowski, en su libro sobre la esquizofrenia, celebra la geometría no-euclidiana, en concreto la geometría hiperbólica de Lobatchevsky. Por otro lado, el contacto de Beckett con el mundo de la psiquiatría supera lo puramente metafórico para concretarse en una vivencia de tipo personal, ya que él mismo fue paciente de los psicoanalistas Geoffrey Thompson y Wilfred Bion. Por recomendación de este último el escritor asistió en I933 a una conferencia de C. G. Jung, que le dejó, según su propio testimonio, profundamente impresionado al comprobar que él mismo compartía ciertos síntomas con algunos enfermos mentales. 55 En esta línea se comprende

52. Eugène Minkowski, Viktor E. von Gebsattel y Erwin W. Straus, Antropología de la alienación, trad. Sofía Elisa Lecca (Caracas: Monte Ávila Editores, 1970).

53. Eugène Minkowski, La esquizofrenia. Psicopatología de los esquizoides y los esquizofrénicos, trad. Ana H. Rose (Buenos Aires: Paidós, 1980), 72.

54. Yann Mével, L'Imaginaire mélancolique de Samuel Beckett, de Murphy à Comment c'est (Ámsterdam y Nueva York: Rodopi, 2008), 279.

55. "Siempre he tenido la impresión de que dentro de mí había un ser asesinado. Asesinado antes de mi nacimiento. Tenía que encontrar a ese ser asesinado. Intentar devolverle la vida [...] 
mejor la relación que muchos autores han establecido entre la obra de Beckett y la esquizofrenia, entre ellos Deleuze y Guattari. ${ }^{56}$ Además, el interés de Beckett por la anomalía psíquica se pone de relieve a lo largo de toda su obra en la piel de sus personajes, especímenes ejemplares de una intrigante alienación. ${ }^{57}$ Basta con volver la mirada a los protagonistas de Esperando a Godot, que devienen individuos literalmente propensos a ser diagnosticados de esquizofrenia:

Pozzo representaría con claridad lo que normalmente consideramos una personalidad no-integrada. De hecho, él y todos los innumerables personajes de Beckett que tanto se parecen a él en este respecto, en mucho se asemejan a los síntomas de un esquizofrénico cuyo psicoanalista Eugène Minkowski describe..$^{58}$

No obstante, Eugène Minkowski no sólo analiza la percepción espacial del sujeto esquizofrénico; también se interesa por su percepción temporal. La obra clave en este campo es El tiempo vivido, basada a su vez en el pensamiento de

Un día fui a escuchar una conferencia de Jung [...] Habló de una de sus pacientes, una chica jovencísima... Al final, mientras la gente se iba marchando, se quedó callado. Y como hablándose a sí mismo, asombrado por el descubrimiento que estaba haciendo, dijo: 'En el fondo no había nacido nunca'. Siempre he tenido la impresión de que yo tampoco había nacido nunca”, Samuel Beckett citado en Charles Juliet, Encuentros con Samuel Beckett, trad. Julia Escobar (Madrid: Siruela, 2006), 20.

56. Esta cuestión ha sido abordada en Mary Bryden, "The schizoid space: Beckett, Deleuze, and L'Épuisé”, en Sjef Houppermans, ed., Beckett \& la psychoanalyse/Beckett \& psychoanalysis, Samuel Beckett Today/Aujourd'hui: 5 (Ámsterdam y Atlanta: Rodopi, 1996), 85-94.

57. Por ejemplo el caso de Molloy: "Molloy es un caso ejemplar de este sujeto esquizofrénico. Se refiere a sí mismo alternativamente en primera o tercera persona, olvida constantemente su propio nombre, y admite 'pavonearse' ante sí mismo 'como un extraño'. Incluso su relación con los objetos y el lenguaje se caracteriza por una ruptura o separación: la separación entre un objeto y su función, entre una palabra y su significado". ("Molloy is exemplary of this schizophrenic subject. He refers to himself alternately in the first person and the third person, consistently forgets his own name, and admits to 'strut[ting]' before himself 'like a stranger.' Even his relationship to objects and to language is characterized by a split or separation: the separation between an object and its function, a word and its meaning", en Sarah Gendron, Repetition, Difference, and Knowledge in the Work of Samuel Beckett, Jacques Derrida, and Gilles Deleuze [Nueva York, Washington, Oxford, Berlín y Viena: Peter Lang, 2008], I42) (trad. de la autora).

58. "Pozzo would clearly represent what we normally consider to be a nonintegrated personality. In fact, he and all the countless Beckett characters who so closely resemble him in this respect, very much mimic the symptoms of a schizophrenic whom psychoanalyst Eugène Minkowski describes", en D. Heyward Brock, ed., The Culture of Biomedicine. Studies in Science and Culture (Newark, Londres y Toronto: Associated University Press, 1984), 192 (trad. de la autora). 
Henri Bergson. En ella Minkowski expone que la mente patológica no concibe el tiempo como una continuidad lineal, es decir, como la sucesión de unos hechos pasados que se encaminan al futuro. Por el contrario, para el esquizofrénico, los eventos se muestran deshilvanados entre sí. Esta alteración se convierte en uno de los principales rasgos de la esquizofrenia, el cual se ha dado en llamar precisamente tiempo entrecortado. Producto de esta percepción patológica, nos encontramos ante un encabalgamiento anómalo de la linealidad temporal, ahora cortocircuitada, ya que para los esquizofrénicos sólo existe una concepción multidimensional del tiempo.

En este contexto, la visión temporal de Giacometti, plasmada en "El sueño, el Sphinx y la muerte de T.”, se presenta muy parecida a ésta que experimentan los esquizofrénicos. Y es que para Giacometti, al igual que para Minkowski - siguiendo a Bergson-, el tiempo no es otra cosa que devenir, una masa desorganizada que discurre, una duración que fluye sin sometimiento al orden racional. No en vano, Minkowski ha señalado acertadamente el carácter irracional de este tipo de temporalidad, erigida en propiedad incontestable de las mentes patológicas. Quizá por eso ha interesado tanto a los artistas surrealistas, y quizá por eso también Giacometti quiso visualizarla en su diagrama en forma de disco. En su superficie Giacometti engarza una miscelánea de vivencias, que retornan al presente revestidas con cierto aire de remordimiento. En los esquizofrénicos, según la definición que aporta Minkowski, esta cualidad se designa "hiperplasia", esto es, una forma de vivir el pasado como evocación y arrepentimiento de algún recuerdo penoso. En definitiva, se trata de otro mecanismo mórbido cuyos efectos distorsionan la unidireccionalidad temporal y alteran así la flecha del tiempo. Sírvanos en este punto el testimonio de un paciente de Minkowski, que por su semejanza bien podría ponerse en boca de Giacometti:

Soy como una flecha ardiente que se arroja hacia adelante, después, se detiene, vuelve hacia atrás y se apaga después como en un espacio vacío en el aire. Es arrojada hacia atrás. Quiero decir con ello que no hay futuro y que soy rechazado hacia atrás. [...] No sé si eran recuerdos que venían del pasado los que se me presentaban, o cómo había sido llevado contra mi voluntad. En todo caso, el pasado se presentaba delante de mí de una forma especialmente impetuosa, pero no como lo veo habitualmente. Lo que, en la vida, se encuentra detrás de nosotros, se adhiere sin embargo todavía a 
nosotros y sabemos cómo ha sucedido todo esto. Cada uno vuelve a encontrarse en su propia persona, ¿no es cierto? Pero yo estaba como truncado de mi propio pasado. 59

Concluimos, pues, con la idea de que la percepción espacio-temporal en Beckett y Giacometti posee una base común, enraizada fundamentalmente en el pensamiento de Bergson, si bien cada uno desarrolla sus propios procedimientos por una vertiente distinta. Como se ha visto, ésta podría abordarse desde la tetradimensionalidad, pero también desde el funcionamiento de la mente esquizofrénica, ya que tanto Beckett como Giacometti demuestran importantes puntos de conexión con la visión espacio-temporal de los esquizofrénicos. Al decir esto, estamos pensando especialmente en una concepción abierta de esquizofrenia, que participa sobre todo de la línea de filiación trazada por el antipsiquiatra Ronald D. Laing, autor de El yo dividido (1964). Esta postura tiende hacia una reivindicación de la figura del esquizofrénico frente a las construcciones teórico-clínicas que subyugan a los alienados (véase el análisis de la locura de Michel Foucault). Entronca finalmente con propuestas de revisión ontológica como la de L'Anti-CEdipe (I972), obra del filósofo Gilles Deleuze y del esquizoanalista Félix Guattari, quienes emprenden una revaloración del esquizo desde el punto de vista de la producción maquínico-deseante. †

59. Minkowski, El tiempo vivido, 268. 